\title{
An Intelligent PID Parameters' Adjustment Algorithm for Control System
}

\author{
Chunqiang Zhu \\ Xi'an Junior Electric Power Technical College, Shanxi Xi'an, 710000 \\ zhuchunqiang@qq.com
}

\begin{abstract}
The parameters' adjustment of Control System is very complicated. Such as, the adjustment of voltage, temperature and liquid level, they have the characteristic of nonlinear and mutation. The traditional PID control algorithm has the defect of overstrike and the low accuracy of adjustment, in order to improve the control performance of Control System, we proposed an improved intelligent PID algorithm for Control System parameters' adjustment. First, we encode the three parameters of PID to a firefly position vector; then, find the optimal PID parameters by simulating the firefly population's food forage and mating behavior; last, adjusted the parameters of PID to intelligent control the Control System. We also using MATLAB toolbox to simulate the proposed algorithm, and give a group of contrast experiments about two control systems, the voltage adjustment and liquid level adjustment. The experiments' results show that contrast to the traditional PID parameters' optimization algorithm, our improved algorithm can quick adjust the Control System along with less overshoot, the response time is faster and the system is more robust.
\end{abstract}

Keywords: PID, Firefly algorithm, control system, parameters adjustment, MATLAB Simulink

\section{Introduction}

With the rapid development of science and technology, many more traditional industries using Control System to control all parameters of the work[1], such as voltage control, liquid level control and temperature control. Nowadays, traditional industry enter into the information times, the demand of robust control for Control System of the traditional industry is increasingly high. And the performance of a Control System depends on the parameters adjustment, so the optimization of parameters' adjustment algorithm has an important significance for the steady running of a Control System.

In the past decade, many researchers lucubrate the research of control optimization problem, and get a certain research achievement. At present, the Control System parameters' adjustment commonly adopt PID (Proportional Integral Differential) accommodation mode [2]. PID controllers give the control effect by proportional, integral and differential, and these three parameters determine the stand or fall of controlling effect. Traditional PID parameters optimization such as "Ziegler-Nichols", "Climbing Algorithm" and "Gradient Method", these algorithms are sensitive to the initial values, the efficiency of optimization searching is slow, so it is hard to find the global optimal PID parameters. With the development of Control System, the optimal problem has the nonlinear character, traditional algorithms are hard to accurate describe the variation trend of parameters. Along with the development of non-linear theory, some researchers' fusion ANN (Artificial Neural Network) and PID to increase the accuracy of controlling [3], but the ANN has a complicated structure and superfluous parameters need to be determine. Besides, other researchers using GA (Genetic Algorithm) and PSO (Particle Swarm 
Optimization) to optimize the PID parameters, to reach a better control effect, these improvements get the corresponding achievement.

The firefly algorithm is a new swarm optimization algorithm that simulate the fireflies' food forage and mating, it has the advantage of simple, strong robustness and efficiency searching optimal parameters ability, extremely appropriate to optimize and control for complex non-linear Control System [4]. In order to improve the accuracy of Control System, this article proposed an improved optimization of PID algorithm to adjust Control System parameters, adopting firefly algorithm to optimize the parameters of PID online, aim at the defect of firefly algorithm, we give some improvements. The simulation results show that our method can improve the performance of Control System.

\section{The Improved Firefly Algorithm}

\subsection{The Traditional Firefly Algorithm}

The firefly algorithm is a random optimization algorithm that construct by simulating the luminescent behavior of fireflies in nature world. The fireflies attracted each other by the luminous intensity, we call it "fluorescent brightness", and the fluorescent brightness of one firefly is determined by the objective function, for every two fireflies, the brighter one will attract the other to close to it. By the continuous renewal of "brightness and attraction degree", the algorithm can achieve the object optimization. The relative fluorescent brightness of one firefly can be defined as:

$$
I=I_{0} e^{-\gamma r_{i j}}
$$

where $I_{0}$ is the maximum of fluorescent brightness for fireflies, the fluorescent brightness of $r=0, \gamma$ is the weight of light intensity sorption, $r_{i j}$ is the space distance between two fireflies $i$ and $j$.

The attraction degree of fireflies can be defined:

$$
\beta=\beta_{0} e^{\gamma_{i j}}
$$

where $\beta_{0}$ is the maximum of attraction degree for fireflies, the attraction degree of $r=0$.

The firefly $i$ is attracted by firefly $j$, and close to it that the updated distance is described by:

$$
x_{i}=x_{j}+\beta\left(x_{j}-x_{i}\right)+\alpha\left(\operatorname{rand}-\frac{1}{2}\right)
$$

where $x_{i}, x_{j}$ are the spatial locations of $i$ and $j$, respectively. $\beta$ is the attraction degree, $\alpha$ is the moving step length, rand is the random numbers between $[0,1]$.

\subsection{The Improved Firefly Algorithm}

In the traditional firefly algorithm, all fireflies need to compared with each other to get the best result, this procedure will lead to a long time consumption for searching the optimization result, so that the algorithm's efficiency is too low, and easy to trap into local minimum. In order to deal with these defects, we improve the traditional firefly algorithm in this article. First, we include two parameters $\xi$ and $\zeta$, defined as:

$$
\begin{aligned}
& \zeta=\bmod [(t-1), \text { gen }] / \text { gen } \\
& \xi=\left(\operatorname{rank}_{i}\right)^{-\zeta}
\end{aligned}
$$


where $t$ is the number of current iterations, gen is the maximum iterations, $\operatorname{rank}_{i}$ is the sorting position of fireflies, $\xi$ is the probability of attracting other fireflies to close to it.

In every iterative procedure, the improved algorithm updates the value of $\xi$ and $\zeta$. When the value of $\xi$ is greater than random number $\tau$, this firefly can attract other fireflies to close to it. Compared with the traditional firefly algorithm, the improved algorithm using parameter $\xi$ to ensure that the movement of one firefly not only relative to attraction degree, but also relative to the threshold $\xi$. So, the movement of fireflies is more rely on the brighter fireflies' attraction degree and the number of iterations.

After that, in order to accelerate the speed of convergence and the global searching ability, we modify the moving step length by stepwise depletion procedure, the detail is as follow:

$$
\alpha=\alpha * \sqrt[g e n]{\lambda}
$$

where the parameter of $\lambda$ is the constant between [0.001 0.01$]$.

In order to test the effectiveness of the improved firefly algorithm in this article, we choose four measurement function to do four groups of contrast simulation experiments, the four standard measure testing function defined as follow [5]:

(1) Sphere function:

$$
f_{1}(x)=\sum_{i=1}^{n} x_{i}^{2}
$$

(2) Rosebrock function:

$$
f_{2}(x)=\sum_{i=1}^{n-1}\left[100\left(x_{i+1}-x_{i}^{2}\right)^{2}+\left(x_{i}-1\right)^{2}\right]
$$

(3) Rastrigin function:

$$
f_{3}(x)=\sum_{i=1}^{n}\left[x_{i}^{2}-10 \cos \left(2 \pi x_{i}\right)+10\right]
$$

(4) Griewank function:

$$
f_{4}(x)=1+\frac{1}{4000} \sum_{i=1}^{n} x_{i}^{2}-\prod_{i=1}^{n} \cos \left(\frac{x_{i}}{\sqrt{i}}\right)
$$

As shown in Figure 1, compared with the traditional standard firefly algorithm(the upper line is traditional firefly algorithm, and the under line is improved firefly algorithm by us), the improved firefly algorithm can avoid trapping into local minimum, it can get higher accuracy and speed up the speed of convergence. The contrast experiment results demonstrate that our improved algorithm has good effectiveness and superiority. 


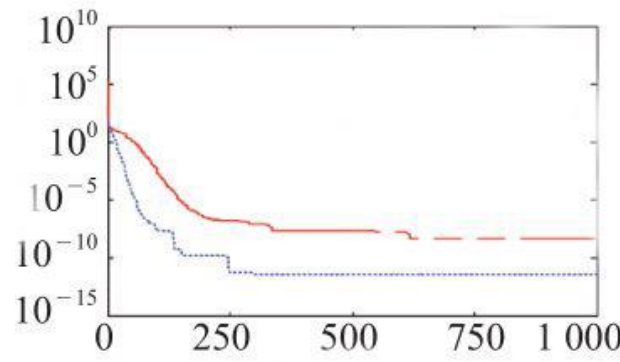

(a) Sphere Function

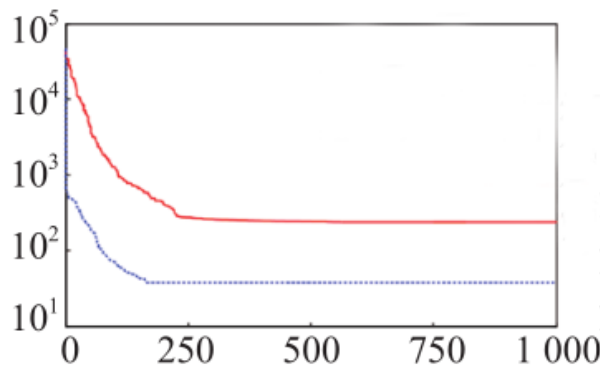

(c) Rastrigin Function

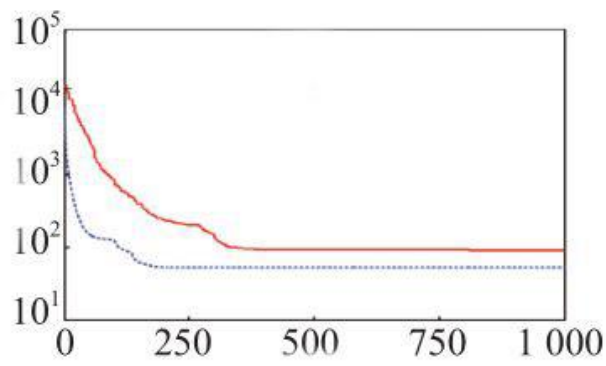

(b) Rosenbrock Function

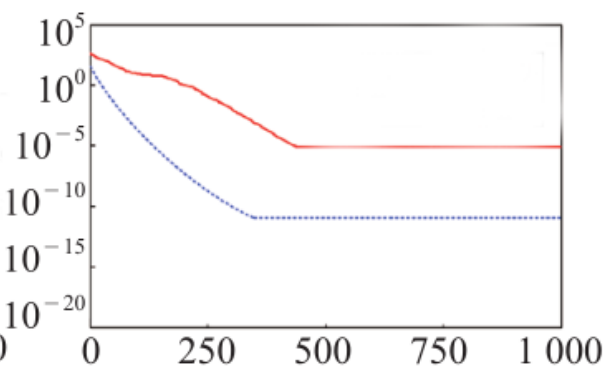

(d) Griewank Function

(The horizontal ordinate is iterations and the vertical ordinate is the value of fitness)

\section{Figure 1. The Four Evaluation Functions of PID Algorithm}

\section{Optimizing PID Parameters in Control System of Voltage Adjuster}

In order to verify our improved algorithm, we do an example of PID parameters' adjustment for voltage [6]. First, we give the mathematical model for voltage adjuster, after that we adopt the improved firefly algorithm to the voltage adjuster, and analyze the results of experiments.

\subsection{The Mathematical Model of Voltage Adjuster}

In an electric power system, the generator transmission function can be described by first order time delay, the detail is as follow:

$$
G_{G}(s)=\frac{K_{g}}{1+T_{d_{0}} s}
$$

where $K_{g}$ is the amplification coefficient of generator, $T_{d_{0}}$ is the time constant and $s$ is the complex variable.

The voltage test unit is used for detecting the output voltage of generator, and it controlled the excitation power, the following transmission function can be defined as:

$$
G_{M}(s)=\frac{K_{r}}{1+T_{r} s}
$$

where $K_{r}$ is the input and output proportion coefficient of voltage sensor, $T_{r}$ is the time constant of filter return circuit.

The main effect of power unit is amplified excitation control signal, is also can be regarded as an inertial element, the corresponding transmission function can be described as:

$$
G_{A}(s)=\frac{K_{a}}{1+T_{a} s}
$$


where $K_{a}$ is the voltage proportion coefficient of power amplified unit, $T_{a}$ is the time constant of amplified unit.

Now, the PID control algorithm for controlling the voltage adjuster can be described as:

$$
u(t)=K_{p} e(t)+K_{i} \int_{0}^{t} e(t) d t+K_{d} \frac{d e(t)}{d t}
$$

In order to get a favorable controlling effect of voltage, the first choice is the best appropriate algorithm of PID (Proportional, Integral and Differential). In our article, we use the improved firefly algorithm to optimize the three parameters from PID controller. This strategy will improve the performance of system.

\subsection{The Coding of Parameters}

Assumed that the whole fireflies in the population is the number of $M$, and every firefly's position vector is made up with the three parameters of PID controller, this means that the vector dimension of firefly is $D=3$, the following matrix can be expressed as:

$$
P(M, D)=\left(\begin{array}{ccc}
K_{p}^{1} & K_{i}^{1} & K_{d}^{1} \\
K_{p}^{2} & K_{i}^{2} & K_{d}^{2} \\
\cdots & \cdots & \cdots \\
K_{p}^{M} & K_{i}^{M} & K_{d}^{M}
\end{array}\right)
$$

\subsection{The Design of Fitness Degree Function}

The objective of PID parameters optimization is used for minimizing the error of controlling for voltage adjuster, and shorting the response time. So we adopt the error absolute value multiple the performance indicator of time integral as the objective function of parameters choice, then we can choose the fitness degree function as:

$$
F(I T A E)=\int_{0}^{\infty} t|e(t)| d t \rightarrow \min
$$

We use the improved firefly algorithm to calculate the optimization value, so we need to deal with the fitness degree function. In the model construction by "Simulink"[7], we regard the reciprocal value of this function as objective function. By this procedure, we transform the minimum objective function to maximum objective function.

\subsection{The Working Principle of Improved Firefly Algorithm Optimize PID Controller}

In the application of improved firefly algorithm on voltage adjuster, we encode the three parameters of PID controller as a position vector of a firefly, and then simulating the firefly in natural world to search a group of optimal parameters, simulate the fireflies' shining behavior and the population action of food forage and mating, the following Figure 2 described the working principle. 


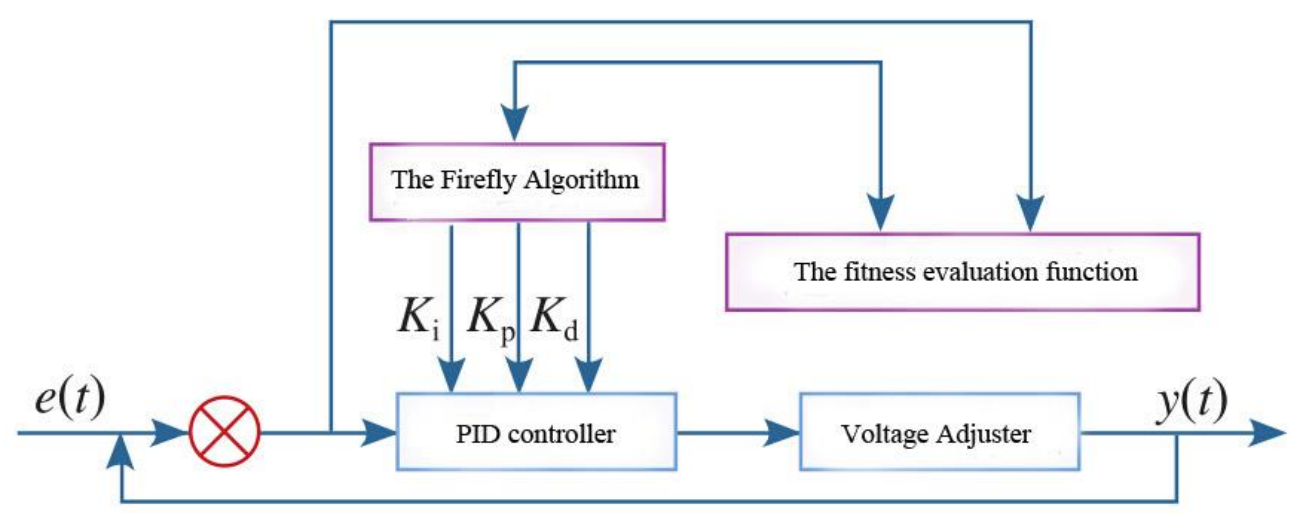

Figure 2. The Working Principle of Optimizing PID Controller by Firefly Algorithm

\subsection{The Steps of Improved Firefly Algorithm Optimizing PID Parameters}

(1) Determine the fireflies' population scale $m$, population dimension $d$, maximum evolution generation iter_max , and the boundary value of controller parameters $K_{p}, K_{i}, K_{d}$.

(2) Random initialize the position of every firefly, the position vector of every firefly is made up with a group of three control parameters from PID controller.

(3) Calculate the fitness value of every firefly, these values are regarded as their maximum fluorescence intensity, and ordered by the value.

(4) According to the formula (2-4), we can calculate the parameters $\xi$ and $\zeta$, then create random number $\tau \in[0,1]$.

(5) According to the formula (2-1), we can calculate the relative fluorescence intensity of every individual firefly, in addition, compared with the adjacent fireflies fluorescence intensity, meanwhile, compared with the value of $\xi$ and $\tau$, this can determine the evolution direction of corresponding firefly.

(6) According to the formula (2-2), we can calculate the attraction degree of every individual firefly, and use the attraction degree result and the formula (2-3) to update the new position of every individual firefly.

(7) Judge if the algorithm iterations reach the setting maximum evolution numberiter_max, if it is, then output the optimal results and the best position, else, command $t=t+1$, and turn to step (2).

\subsection{Simulation Experiments and Results Analysis}

In order to validate the feasibility and the efficiency of our improved firefly algorithm, apply to optimize the PID parameters adjustment in Control System, Voltage Adjuster. We build a simulation experiment environment and the corresponding simulation conditions, after experiments we analyze the results.

We do a group of experiments on the simulation system of voltage adjuster. In the experiments' initialization, we set the firefly population as 100 , and the dimension of controlling parameters as 3 , the maximum iterations as 200 . As a contrast, we do the same experiments using Z-N algorithm to compare with our improved algorithm [8], mainly simulate on the generator's excitation and disturbance state, the system input using single phase step. 
After contrast experiments, we get the excitation response time curve between our improved algorithm and Z-N algorithm, Figure 3 shows the results. The device voltage response time of simulative voltage that produce 30 percent disturbance in 4 seconds is shown in Figure4. From the results in Figure 3(a), (b), we can research, in the iteration time, the time consumption of adjustment in our improved algorithm for generator voltage excitation and disturbance are shorter, the time complexity is prominent better than Z-N algorithm, and it can obtain more perfect controlling effects. Besides, Figure 3(c) gives the contrast results of module voltage output wave form. From the result, we can research that the fitting precision of our improved algorithm is better than Z-N algorithm. Adopt the improved firefly algorithm to optimize the PID parameters adjustment is the main reason, this procedure can implement intelligent control the voltage, reduce a lot of fitting errors.

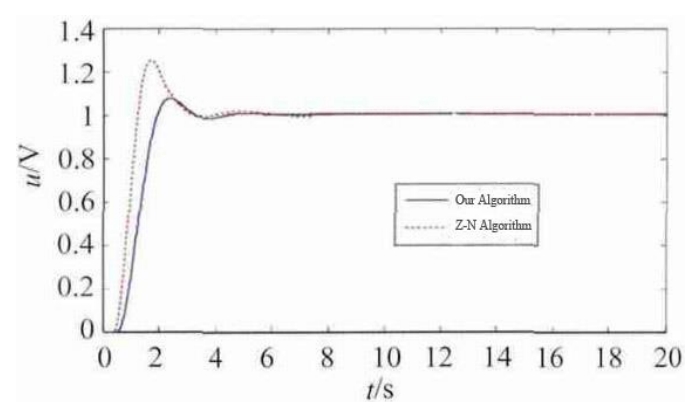

(a) Voltage Boost Curve of Two Algorithms

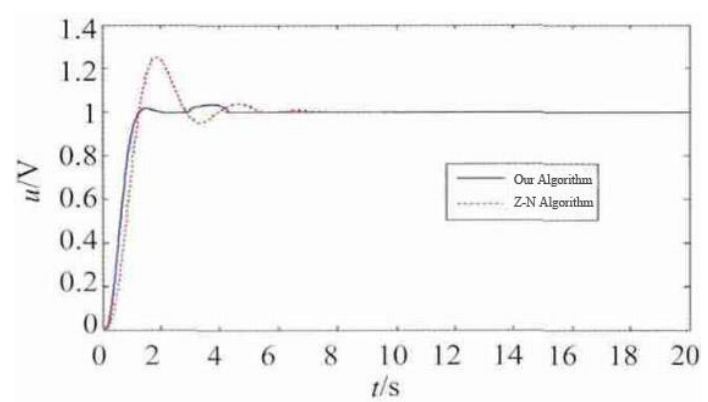

(b) Load Voltage Disturbance of Two Algorithms

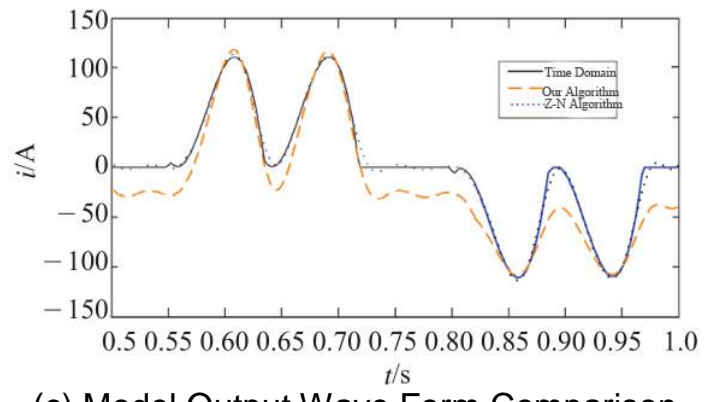

(c) Model Output Wave Form Comparison

Figure 3. Experiments and Results of Two Algorithms

\section{Optimizing PID Parameters in Control System of Liquid Level}

Liquid level control system belong to supervisory computer control system[9], using computer to control the discrete objects sampled in the liquid level control system, it also use the PID control algorithm to execute the liquid level output, so that it can implement constant liquid level control in control system. In this chapter, we apply our improved algorithm to realize the liquid level control in a Control System.

\subsection{Liquid Level Control Mathematical Model}

Figure 4 gives the structure chart of a liquid level control system, this system adopts closed cycle control scheme. The A/D data capture card make the actual liquid level output sampled to discrete data, the discrete output data then feedback to the input port. Digital regulator adopts intelligent PID algorithm to adjust the parameters, output the discrete signal, by D/A transfer cards. Then transmit to simulation control electrical level signal, the signal can control water aspirator to control the actual liquid level, this is like the voltage control for voltage adjuster as before. 


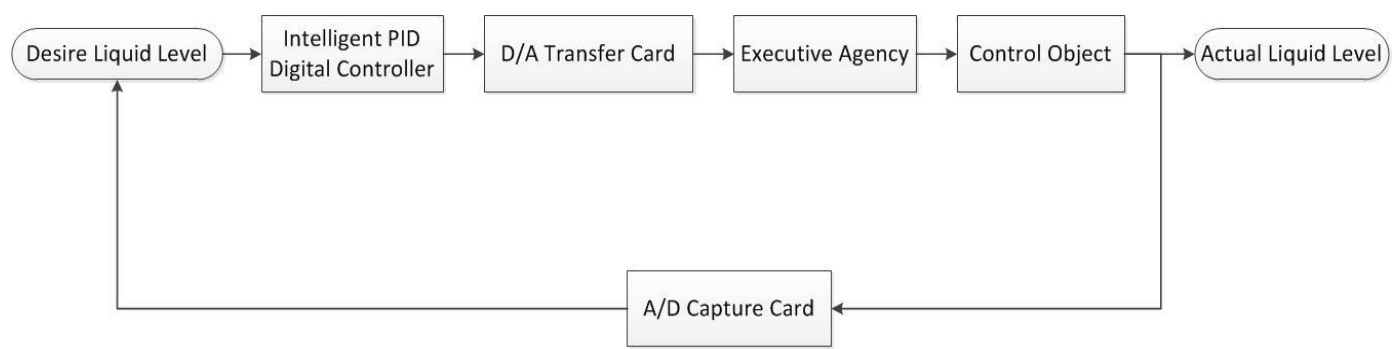

Figure 4. The Structure Chart of a Liquid Level Control System

\subsection{The Improved PID Algorithm used for Control Liquid Level}

In the control system of liquid level, due to the liquid level has the character of large lag, nonlinear, and the mathematical is complex, the traditional PID algorithm will appear large overstrike phenomenon, the dynamics of system is poor, it is difficult to reach satisfactory results. So, we adopt our improved intelligent PID algorithm, using firefly algorithm to adjust PID parameters, this improved algorithm will deal with the positive characters, such as large lag, nonlinear and time various.

When the system output error absolute value is so large, the control system needs to adopt saturated-output work mode, this strategy can reduce the time lag of liquid level control system. Meanwhile, in order to prevent the system come to a large overstrike value, adopt increasing integral coefficient method when the system's error absolute value in a small range, this will increase the precision of steady state. Our improved intelligent PID algorithm based on firefly algorithm to adjust parameters that can answer this positive characters, it can prominent improve the dynamic response and steady state precision of liquid level control system.

The control regulations of intelligent PID algorithm are defined as follow:

Regulation 1. If $\left|e(k)>e_{\max }\right|$, then

$$
u(k)=u_{\max }(k)
$$

Regulation 2. If $|e(k)|<e_{\text {min }}$, then

$$
u(k)=u(k-1)
$$

Regulation 3. If $e_{\min }<|e(k)|<e_{\max }$, then

$$
u(k)=u(k-1)+k_{p 1} \times[e(k)-e(k-1)]+k_{i 1} \times e(k)
$$

Regulation 4. If $e_{\min }<|e(k)|<e_{\text {mid }}$, then

$$
u(k)=u(k-1)+k_{p 2} \times[e(k)-e(k-1)]+k_{i 2} \times e(k)
$$

where $e(k)$ is the deviation, $u(k)$ is the controlled quantity, $e_{\min }$ is the minimum permissible deviation, $e_{\max }$ is the maximum permissible deviation, $e_{\text {mid }}$ is the middle permissible deviation, $u_{\max }(k)$ is the maximum controlled quantity, $k_{p 1}, k_{p 2}, k_{i 1}, k_{i 2}$ is the controlling weight of deviation in intelligent algorithm, and they are satisfied: $k_{p 1}>k_{p 2}, k_{i 1}>k_{i 2}$.

According to the four regulations above, we can find that our improved intelligent algorithm is essentially nonlinear that can excellent overcome the defect of traditional 
PID control algorithm. Regulation 1, 2 show the rapidity and stability of control system, regulation 3, 4 show the parameters adjustment by intelligent algorithm.

\subsection{Simulation Experiments and Results Analysis}

In order to validate the feasibility and the efficiency of our improved firefly algorithm, apply to optimize the PID parameters adjustment in Control System, Liquid Level. We build a simulation experiment environment and the corresponding simulation conditions, after experiments we analyze the results.

We do a group of contrast experiments between traditional algorithm (PID and BP algorithm) and our improved algorithm (we call PIDFF algorithm). The experiments are built on the MATLAB platform with "Simulink". Figure 5(a) (b) (c) give the traditional PID control algorithm results (PID, BP [10]) and the improved intelligent PID control algorithm (PIDFF) results, respectively. And the table 1shows the three algorithms' performance index in liquid level control system, we record the overstrike value, adjust time and ascend time three indexes.

Table 1. Phase Step Input Condition of the Control System Performance Index

\begin{tabular}{|l|l|l|l|}
\hline Control Algorithm & Overstrike Value/\% & Adjust Time/s & Ascend Time/s \\
\hline PID & 39 & 63 & 5 \\
\hline BP & 17 & 52 & 11 \\
\hline PIDFF & 8 & 32 & 4 \\
\hline
\end{tabular}
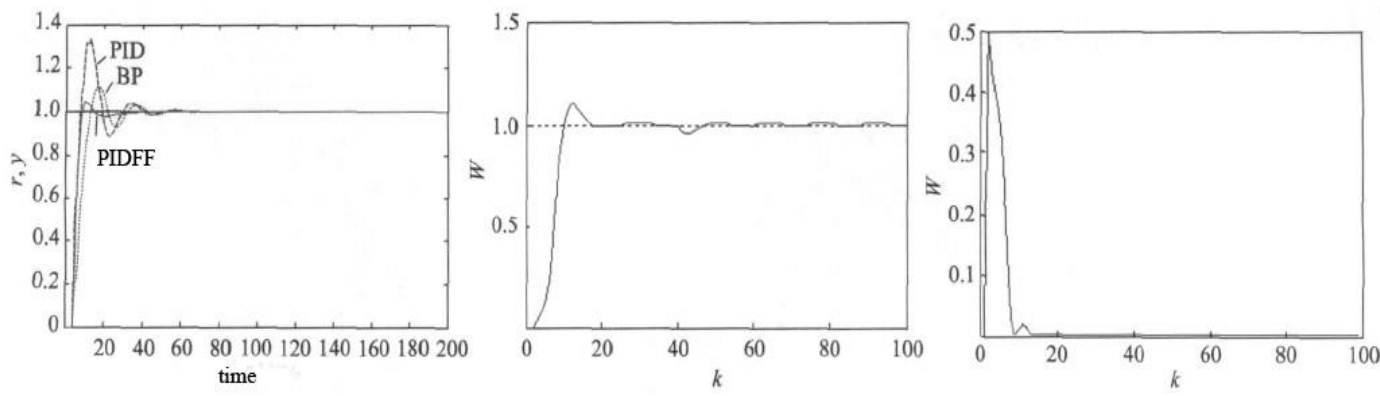

(a)Liquid Level Phase Step Response (b) Phase Step Response Curve (c) Object Function Curve

Figure 5. Experiments and the Results of Three Algorithms

From Figure 5 and Table 1, we can research that compared to the traditional PID algorithm, our improved PIDFF algorithm lead the system control time reducing 31 seconds, and the overstrike value's reduction is more remarkable(79.5\%), dramatically increased control performance. Compared to another PID improved algorithm optimized by BP neural network, the system reduce 20 seconds, and the overstrike value's reduction is about $\mathbf{5 2 . 9 \%}$, the performance is remarkable. PIDFF's ascend time is also short, this means that our improved algorithm have a better response speed than other algorithms.

\section{Conclusion}

In this article, we propose a new algorithm for optimizing the PID parameters' adjustment by firefly algorithm. First, we introduce the theory of PID in control system, and analyze the defect of traditional PID algorithm as follow, then we introduce the firefly algorithm to dynamic optimize the PID parameters, and we give the detail steps of optimization procedure. In order to validate our proposed algorithm in control system, we give two examples of control system, the voltage adjustment and the liquid level 
adjustment. In these two examples, they have some common grounds and some differences, so we use these two control system model to validate our algorithm. We do a group of contrast experiments about two models, and use MATLAB "Simulink" to simulate, the results of two experiments show that our improved algorithm has better ability in control system, and it has high precision and high efficiency than traditional PID algorithm. Therefore, our algorithm can make a good contribution in Control System.

\section{References}

[1] B. Jayalakshmi, V. R. Pramod, "Total Interpretive Structural Modeling (TISM) of the Enablers of a Flexible Control System for Industry”, Global Journal of Flexible Systems Management, vol. 16, no. 1, (2015), pp. 63-85.

[2] T. Zhang, Y. Jin and Y. A. Ding, "Cytokine network-inspired cooperative control system for multi-stage stretching processes in fiber production", International Journal of Soft Computing, vol. 19, no. 6, (2014), pp. $1523-1540$.

[3] V. Kumar, P. Gaur and A. P. Mittal, "ANN based self-tuned PID like adaptive controller design for high performance PMSM position control”, Expert Systems with Applications (journal), vol. 41, no. 17, (2014), pp. 7995-8002.

[4] P. Dash, L. C. Saikia and N. Sinha, "AGC of a multi-area system using firefly optimized two degree of freedom PID controller", Power and Energy Systems Conference: Towards Sustainable Energy, IEEE, 2014, (2014), pp. 1-6

[5] I. Fister, S. Fong and J. Brest, "A novel hybrid self-adaptive bat algorithm", The Scientific World Journal, (2014).

[6] S. Malladi, K. P. Swaroop and O. Koduri, "Loop slope adjustment methods for the control of Automatic Voltage Regulator", Green Computing Communication and Electrical Engineering (ICGCCEE), 2014 International Conference, IEEE, (2014), pp. 1-6.

[7] M. M. Bello, A. A. Shafie and M. R. Khan, "Electro-Hydraulic PID Force Control for Nonlinear Vehicle Suspension System", International Journal of Engineering Research and Technology, ESRSA Publications, (2015), p. 4.

[8] M. J. Neath, A. K. Swain and U. K. Madawala, "An optimal PID controller for a bidirectional inductive power transfer system using multi-objective genetic algorithm”, Power Electronics, IEEE, vol. 29, no. 3, (2014), pp. 1523-1531.

[9] F. Ma, "An improved fuzzy PID control algorithm applied in liquid mixing system", Information and Automation (ICIA), 2014 IEEE International Conference, IEEE, (2014), pp. 587-591.

[10] W. Kang, T. Chu, H. Zhao, "The Design of Constant Tension System Based on BP Neural Network PID Control”, Scientific Journal of Control Engineering, vol. 4, no. 2, (2014).

\section{Author}

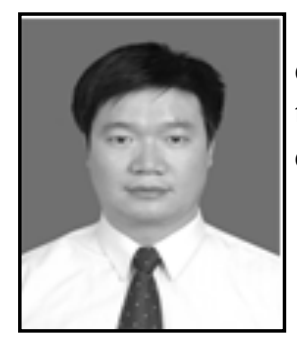

Chunqiang Zhu, he has a research major in control science and data mining, proficient in using intelligent algorithms to deal with the traditional Control System, have some experience about industrial control system. 